\title{
含腙结构单元的喹唑啉酮衍生物的设计、合成与生物活性研究
}

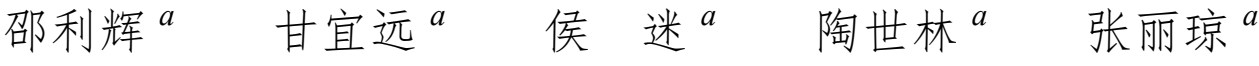 \\ 王贞超 $*, a, b, c$ 欧阳贵平 $*, a, b, c, d$ \\ ( ${ }^{a}$ 贵州大学药学院 贵阳 550025) \\ $(b$ 贵州医科大学药用植物功效与利用国家重点实验室＼cjkstart贵阳 550014) \\ ( 贵州大学精细化工研究开发中心 贵阳 550025) \\ ( 贵州省合成药物工程实验室 贵阳 550025)
}

\begin{abstract}
摘要 以静红酸䣶为起始原料, 设计并合成了一系列新颖的含腙结构单元的喹唑啉酮类衍生物. 所有目标化合物经 ${ }^{1} \mathrm{H}$ $\mathrm{NMR} 、{ }^{13} \mathrm{C}$ NMR 和高分辨质谱(HRMS)表征确证其结构. 初步抗菌活性结果显示, 该类化合物对水稻白叶枯病菌 (Xanthomonas oryzae pv. Oryzae, Xoo)、猕猴桃溃疡病菌(Pseudomonassyringae pv. actinidae, Psa) 和柑橘溃疡病菌 (Xanthomonas axonopodis pv. Citri, Xac)均表现出一定的抑制活性. 其中 3-甲基-2-(((4-((2-(4-甲基苯磺酰基)肼基)甲基) 苯氧基)甲基)喹唑啉-4 $(3 H)$-酮 $\left(\mathbf{G}_{18}\right) 、 3$-甲基-2-(((4-((2-(2,6-二氯苯基)肼基)甲基)苯氧基)甲基)喹唑啉-4 $(3 H)$-䣳 $\left(\mathbf{G}_{12}\right)$ 和 3甲基-2-(((4-((2-(苯磺酰基)肼基)甲基)苯氧基)甲基)喹唑啉-4(3H)-䣳 $\left(\mathbf{G}_{16}\right)$ 对 Xoo、Psa 和 Xac 三种细菌的抑制活性分别优 于对照药叶枯唑和噻菌铜. 另外, 3-甲基-2-(((4-((2-(3,5-二氯苯基)肼基)甲基)苯氧基)甲基)喹唑啉-4(3H)-酮( $\mathbf{G}_{5}$ )对 Xoo、

$P s a$ 和 $X a c$ 三种细菌均表现出良好的广谱抗菌活性.
\end{abstract}

关键词＼cjkstart喹唑啉酮衍生物; 腙; 抑菌活性; 构效关系

\section{Design, Synthesis and Biological Activity of Quinazolinone Derivatives Containing Hydrazone Structural Units}

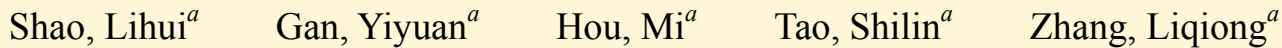 \\ Wang, Zhenchao ${ }^{*, a, b, c} \quad$ Ouyang, Guiping ${ }^{*, a, b, c, d}$ \\ ( ${ }^{a}$ College of Pharmacy, Guizhou University, Guiyang 550025) \\ ( ${ }^{b}$ State Key Laboratory of Functions and Application of Medicinal Plants, Guizhou Medicinal University, Guiyang 550014) \\ ( ${ }^{c}$ Center for Research of Fine Chemicals, Guizhou University, Guiyang 550025) \\ ( ${ }^{d}$ Guizhou Engineering Laboratory for Synthetic Drugs, Guizhou University, Guiyang 550025)
}

\begin{abstract}
A series of novel quinazolinone derivatives containing hydrazone structural units were designed and synthesized with isatoic anhydride as the starting material. All target compounds were characterized by ${ }^{1} \mathrm{H} N \mathrm{NR},{ }^{13} \mathrm{C}$ NMR and HRMS. The preliminary antibacterial activity results showed that the compounds exhibited a certain inhibitory activity against Xanthomonas oryzae pv. oryzae (Xoo), Pseudomonas syringae pv. actinidiae (Psa) and Xanthomonas axonopodis pv. citri (Xac). Among them, $(E)$-4-methyl- $N^{\prime}$-(4-((3-methyl-4-oxo-3,4-dihydroquinazolin-2-yl)methoxy)benzylidene)benzenesulfonohydrazide $\left(\mathbf{G}_{\mathbf{1 8}}\right),(E)$-2-((4-((2-(2,6-dichlorophenyl)hydrazono)methyl)phenoxy)methyl)-3-methylquinazolin-4(3H)-one $\left(\mathbf{G}_{\mathbf{1 2}}\right)$ and $(E)$ $N^{\prime}$-(4-((3-methyl-4-oxo-3,4-dihydroquinazolin-2-yl)methoxy)benzylidene)benzenesulfonohydrazide $\left(\mathbf{G}_{\mathbf{1 6}}\right)$ displayed better antibacterial activity against Xoo, Xac and $P s a$ than the control drugs of bismerthiazol and thiediazole-copper, respectively. Notably, (E)-2-((4-((2-(3,5-dichlorophenyl)hydrazono)methyl)phenoxy)methyl)-3-methylquinazolin-4(3H)-one $\left(\mathbf{G}_{\mathbf{5}}\right)$ displayed fine broad-spectrum antimicrobial activity against Xoo, Xac and $P s a$.

Keywords quinazolinone derivative; hydrazone; antibacterial activity; structure-activity relationship
\end{abstract}

\footnotetext{
* Corresponding authors. E-mail: wzc.4884@163.com; oygp710@163.com

Received March 5, 2020; revised April 10, 2020; published online May 7, 2020.

Project supported by the National Natural Science Foundation of China (No. 21867004), the State Key Laboratory of Functions and Applications of Medicinal Plants (No. FAMP201707K) and the Guizhou Provincial Science Technology Program for Platform and Talents (No. 20185781).

国家自然科学基金(No. 20161055)、药用植物功效与利用国家重点实验室(No. FAMP201707K)和贵州省科技计划(No. 20185781)资助项目.
} 
植物病原菌如水稻白叶枯病菌(Xanthomonas oryzae pv. Oryzae, Xoo)、猕猴桃溃疡病菌(Pseudomonassyringae pv. actinidae, Psa) 和柑橘溃疡病菌(Xanthomonas axonopodis pv. Citri, Xac), 每年对农业生产造成巨大的损失. 以水稻白叶枯病菌为例, 其通过侵害水稻输管系统和木 质部组织引起水稻细菌性枯萎病, 造成水稻减产或枯

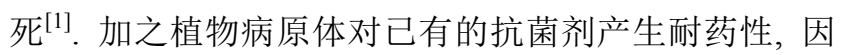
此发现新的高效、低毒、克服耐药性的药物小分子显得 尤为重要.

喹唑啉酮及其衍生物作为重要的药效团, 具有广泛 的药理活性 ${ }^{[2]}$, 如抗菌药氟喹唑(Fluquinconazole $)^{[3]}$ 、抗 肿瘤药诺拉曲特 (Nolatrexed) ${ }^{[4]}$ 和雷替曲塞 (Raltitere$\mathrm{xed})^{[5]}$ 、肌松药氟喹酮(Afloqualone) $)^{[6]}$ 、镇静药安眠酮 (Methaqualone) ${ }^{[7]}$ 等. 喹唑啉酮具有优良的抗菌活性, 一 直是药物合成化学家们关注的热点之一. 2016 年, Sojitra 等 ${ }^{[8]}$ 合成了一系列磺酰胺喹唑啉酮类衍生物, 部分化合 物表现出优异的抗菌活性, 且抗细菌活性优于抗真菌活 性. 2018 年, $\mathrm{Li}$ 等 ${ }^{[9]}$ 设计合成了一系列含有吲哚结构的 喹唑啉酮腙类化合物, 活性测试结果表明, 部分化合物 对 Xoo 和 Xac 的抑制活性明显优于阳性对照药叶枯唑.

此外, 腙类结构因含有 $\mathrm{NHN}=\mathrm{CH}$ 结构, 具有较强 的配位能力、多样的配位方式和特殊的生物活性 ${ }^{[10]}$. 许 多抗菌农药小分子中都含有此类结构片段, 如杀菌剂醌

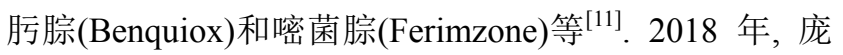
富华等 ${ }^{[12]}$ 设计合成了丹皮酚腙类衍生物, 大部分化合 物对金黄色葡萄球菌和大肠杆菌表现出较好的抑菌活 性, 特别是化合物 I 对金黄色葡萄球菌的最低抑菌浓度 (MIC)为 $2 \mu \mathrm{g} \cdot \mathrm{mL}^{-1}$, 优于阳性对照药环丙沙星.

为获得具有更高抗菌活性的喹唑啉酮类化合物, 采 用活性拼接原理, 结合本课题组前期相关研究基础 ${ }^{[13]}$, 以靛红酸酐为起始原料, 在喹唑啉酮母环 2-位通过亚甲 基氧基苯引入腙类结构单元(Scheme 1), 设计并合成了

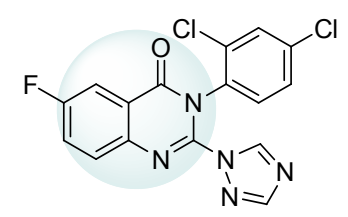

氟喹唑(Fluquinconazole)

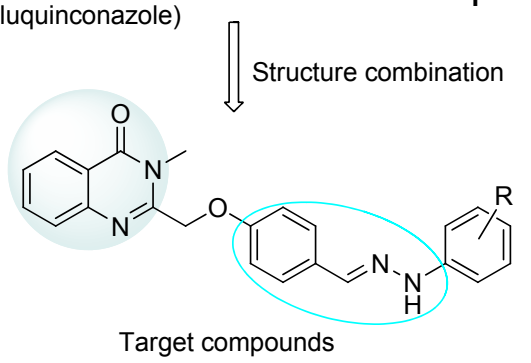

图式 1 目标化合物的设计思想

\section{列}

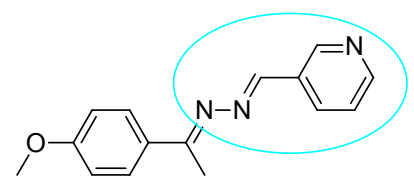


化合物的 ${ }^{1} \mathrm{H}$ NMR 和 HRMS. 在 ${ }^{1} \mathrm{H}$ NMR 谱图中, $\delta$ 10.52 出现的单峰归属于氧基苯环和亚胺中间氮( $\mathrm{NH}-$ $\mathrm{N}=$ )上的活泼氢, $\delta 7.85$ 出现的单峰归属于苯环和亚胺 $(\mathrm{ArCH}=\mathrm{N})$ 相连的亚甲基上的活泼氢, $\delta 8.12 \sim 7.09$ 之间 (除了 $\delta 7.85$ )为喹唑啉酮母环和取代芳香环归属的 12 个 氢, $\delta 5.30$ 出现的单峰归属于酚羟基和喹唑啉酮母环 $\left(\mathrm{ArCH}_{2} \mathrm{O}\right)$ 相连的亚甲基上的氢, $\delta 3.59$ 出现的单峰归属 于喹唑啉酮母环 3-位 $\left(\mathrm{NCH}_{3}\right)$ 氮甲基上的氢. 在 HRMS 谱中, 目标化合物的 HRMS $[\mathrm{M}+\mathrm{H}]^{+}$的测量值与理论值 一致, 在误差允许范围内 $(m / z \pm 0.0030)$, 进一步证明了 化合物的结构.

\section{3 目标化合物的生物活性测试}

\subsection{1 化合物抑菌活性测试}

目标化合物抑菌活性测试结果(表 1)表明，该类化 合物对水稻白叶枯病菌、烟草青枯病菌和柑橘溃疡病菌 均具有一定的抑制作用. 在浓度为 $50 \mu \mathrm{g} / \mathrm{mL}$ 浓度下, $\mathbf{G}_{1} 、 \mathbf{G}_{5} 、 \mathbf{G}_{18}$ 化合物对 Xoo 的抑制率均超过阳性对照药 噻菌铜 (40.0\%), 其中化合物 $\mathbf{G}_{\mathbf{1 8}}$ 抑制率均高于噻菌铜和 叶枯唑 (74.1\%). 在 $50 \mu \mathrm{g} / \mathrm{mL}$ 浓度下, 苯环 3-位引入较 大的官能团对提高化合物对 Xoo 的抑制活性有利, 如化 合物 $\mathbf{G}_{\mathbf{1}}\left(3-\mathrm{NO}_{2}\right)$ 对 Xoo 的抑制活性大于 $\mathbf{G}_{\mathbf{9}}\left(3-\mathrm{CF}_{3}\right) 、 \mathbf{G}_{\mathbf{4}}$ (3-CN)的抑制活性；2-位取代基的变化对活性影响较小, 如 $\mathbf{G}_{7}\left(2-\mathrm{NO}_{2}\right) 、 \mathbf{G}_{\mathbf{8}}\left(2-\mathrm{CH}_{3}\right)$ 和 $\mathbf{G}_{\mathbf{1 4}}\left(2-\mathrm{CF}_{3}\right)$ 三个化合物对 三种植物病原菌的抑制活性相当. 在 $50 \mu \mathrm{g} / \mathrm{mL}$ 浓度下,
$\mathbf{G}_{\mathbf{5}} 、 \mathbf{G}_{7} 、 \mathbf{G}_{\mathbf{8}} 、 \mathbf{G}_{\mathbf{1 0}} 、 \mathbf{G}_{\mathbf{1 2}} 、 \mathbf{G}_{\mathbf{1 6}} 、 \mathbf{G}_{\mathbf{1 8}}$ 化合物对 $P s a$ 的抑制 率, 均超过或接近阳性对照药叶枯唑 $(40.5 \%)$; 在苯环 2位引入供电子基团有利于活性的提高, 如 $\mathbf{G}_{8}\left(2-\mathrm{CH}_{3}\right)$ 对 $P s a$ 的抑制率大于 $\mathbf{G}_{7}\left(2-\mathrm{NO}_{2}\right)$ 和 $\mathbf{G}_{\mathbf{1 4}}\left(2-\mathrm{CF}_{3}\right)$ 的抑制活性. 苯环 3-位和 5-位引入二取代基, 比 2-位和 5-位引入二取 代基活性较好, 如在 $50 \mu \mathrm{g} / \mathrm{mL}$ 浓度下, $\mathbf{G}_{5}\left(3,5-\mathrm{Cl}_{2}\right)$ 对 $X o o 、 P s a$ 和 $X a c$ 的抑菌活性, 均大于 $\mathbf{G}_{6}\left(2,5-\mathrm{Cl}_{2}\right)$. 在 50 $\mu \mathrm{g} / \mathrm{mL}$ 浓度下，化合物 $\mathbf{G}_{5} 、 \mathbf{G}_{7} 、 \mathbf{G}_{\mathbf{1 0}} 、 \mathbf{G}_{\mathbf{1 1}} 、 \mathbf{G}_{\mathbf{1 3}} 、 \mathbf{G}_{15}$ 、 $\mathbf{G}_{\mathbf{1 6}}$ 和 $\mathbf{G}_{17}$ 对 $X a c$ 的抑制率均超过阳性对照药叶枯唑 (41.7\%).

\subsection{2 化合物的抑植物病菌 $\mathrm{EC}_{50}$ 值活性测试}

以上市药叶枯唑和噻菌铜为阳性对照药, 测试部分 化合物对水稻白叶枯病菌、猕猴桃溃疡病菌和柑橘溃疡 病菌的 $\mathrm{EC}_{50}$ 值, 结果列于表 2 4. 由表 2 可知, 所测目 标化合物 $\mathbf{G}_{\mathbf{1}} 、 \mathbf{G}_{5} 、 \mathbf{G}_{\mathbf{1 8}}$ 对 $X o o$ 的 $\mathrm{EC}_{50}$ 值分别为 65.7 、 43.8 和 $5.34 \mu \mathrm{g} / \mathrm{mL}$, 优于对照药噻菌铜 $(69.3 \mu \mathrm{g} / \mathrm{mL})$, 其 中 $\mathbf{G}_{18}$ 的 $\mathrm{EC}_{50}$ 值均优于叶枯唑 $(26.5 \mu \mathrm{g} / \mathrm{mL})$ 和噻菌铜. 由表 3 可知, 所测目标化合物 $\mathbf{G}_{\mathbf{5}} 、 \mathbf{G}_{7} 、 \mathbf{G}_{\mathbf{8}} 、 \mathbf{G}_{\mathbf{1 0}} 、 \mathbf{G}_{\mathbf{1 2}}$ 、 $\mathbf{G}_{\mathbf{1 6}}$ 和 $\mathbf{G}_{\mathbf{1 8}}$ 对 $P s a$ 的 $\mathrm{EC}_{50}$ 值分别为 78.2、69.3、76.2、61.4、 43.4、121 和 $76.4 \mu \mathrm{g} / \mathrm{mL}$, 优于对照药叶枯唑(135 $\mu \mathrm{g} / \mathrm{mL}$ ). 由表 4 可知, 所测目标化合物 $\mathbf{G}_{5} 、 \mathbf{G}_{7} 、 \mathbf{G}_{10}$ 、 $\mathbf{G}_{\mathbf{1 1}} 、 \mathbf{G}_{\mathbf{1 3}} 、 \mathbf{G}_{\mathbf{1 6}}$ 和 $\mathbf{G}_{\mathbf{1 7}}$ 对 $X a c$ 的 $\mathrm{EC}_{50}$ 值分别为 63.6、67.0、 $54.0 、 61.0 、 29.3$ 和 $67.0 \mu \mathrm{g} / \mathrm{mL}$, 优于对照药叶枯唑 $(72.0$ $\mu \mathrm{g} / \mathrm{mL})$.

表 1 目标化合物 $\mathbf{G}_{\mathbf{1}} \sim \mathbf{G}_{\mathbf{1 8}}$ 的抑菌活性(抑制率 $\left./ \%\right)^{a}$

Table 1 Antibacterial activities (inhibition rate/\%) of target compounds $\mathbf{G}_{\mathbf{1}} \sim \mathbf{G}_{\mathbf{1 8}}$

\begin{tabular}{|c|c|c|c|c|c|c|}
\hline \multirow{2}{*}{ Compd. } & \multicolumn{2}{|c|}{$X o o$} & \multicolumn{2}{|c|}{$P s a$} & \multicolumn{2}{|c|}{$X a c$} \\
\hline & $50 \mu \mathrm{g} / \mathrm{mL}$ & $12.5 \mu \mathrm{g} / \mathrm{mL}$ & $50 \mu \mathrm{g} / \mathrm{mL}$ & $12.5 \mu \mathrm{g} / \mathrm{mL}$ & $50 \mu \mathrm{g} / \mathrm{mL}$ & $12.5 \mu \mathrm{g} / \mathrm{mL}$ \\
\hline$G_{1}$ & $41.1 \pm 0.5$ & $13.0 \pm 4.3$ & $36.8 \pm 6.8$ & $22.5 \pm 3.5$ & $22.4 \pm 1.9$ & $19.4 \pm 7.7$ \\
\hline $\mathbf{G}_{2}$ & $27.3 \pm 6.1$ & $22.4 \pm 0.4$ & $28.5 \pm 1.8$ & $26.9 \pm 3.3$ & $36.2 \pm 3.9$ & $19.8 \pm 0.7$ \\
\hline $\mathbf{G}_{3}$ & $28.3 \pm 4.9$ & $23.1 \pm 5.5$ & $27.4 \pm 3.4$ & $24.4 \pm 0.8$ & $24.8 \pm 7.8$ & $20.4 \pm 2.0$ \\
\hline $\mathbf{G}_{4}$ & $26.3 \pm 2.4$ & $23.5 \pm 2.8$ & $38.4 \pm 7.0$ & $21.9 \pm 4.9$ & $24.9 \pm 6.7$ & $14.9 \pm 3.4$ \\
\hline $\mathbf{G}_{5}$ & $56.7 \pm 9.5$ & $24.0 \pm 2.9$ & $46.1 \pm 8.1$ & $31.8 \pm 2.4$ & $44.7 \pm 1.7$ & $12.3 \pm 6.0$ \\
\hline $\mathbf{G}_{6}$ & $35.7 \pm 7.3$ & $19.9 \pm 3.8$ & $35.4 \pm 1.9$ & $26.8 \pm 1.2$ & $26.9 \pm 7.0$ & $12.4 \pm 1.5$ \\
\hline $\mathbf{G}_{7}$ & $25.6 \pm 3.5$ & $21.8 \pm 3.5$ & $42.3 \pm 4.1$ & $13.5 \pm 5.4$ & $44.1 \pm 4.8$ & $23.6 \pm 4.2$ \\
\hline $\mathbf{G}_{8}$ & $24.6 \pm 3.6$ & $24.0 \pm 7.6$ & $46.5 \pm 4.1$ & $31.3 \pm 6.9$ & $18.0 \pm 1.5$ & $14.2 \pm 1.8$ \\
\hline $\mathbf{G}_{9}$ & $29.6 \pm 1.1$ & $27.8 \pm 3.2$ & $38.6 \pm 4.4$ & $24.2 \pm 4.6$ & $31.5 \pm 0.3$ & $20.3 \pm 8.0$ \\
\hline $\mathbf{G}_{10}$ & $21.3 \pm 5.8$ & $15.4 \pm 3.9$ & $38.9 \pm 7.3$ & $14.0 \pm 6.5$ & $46.7 \pm 4.4$ & $18.6 \pm 8.7$ \\
\hline $\mathbf{G}_{11}$ & $31.6 \pm 4.0$ & $29.0 \pm 2.6$ & $33.8 \pm 1.2$ & $20.5 \pm 3.6$ & $45.0 \pm 6.8$ & $27.4 \pm 7.8$ \\
\hline $\mathbf{G}_{12}$ & $22.7 \pm 5.5$ & $19.4 \pm 6.5$ & $52.4 \pm 5.3$ & $21.1 \pm 7.1$ & $32.4 \pm 3.7$ & $21.2 \pm 6.1$ \\
\hline $\mathbf{G}_{13}$ & $24.5 \pm 4.5$ & $20.8 \pm 2.2$ & $29.0 \pm 6.4$ & $25.1 \pm 5.4$ & $45.3 \pm 5.3$ & $25.6 \pm 7.7$ \\
\hline $\mathbf{G}_{14}$ & $24.8 \pm 5.0$ & $12.8 \pm 6.4$ & $36.5 \pm 8.8$ & $25.3 \pm 4.8$ & $31.0 \pm 2.1$ & $22.4 \pm 5.7$ \\
\hline $\mathbf{G}_{15}$ & $36.5 \pm 2.6$ & $33.6 \pm 2.3$ & $35.8 \pm 2.5$ & $20.8 \pm 0.8$ & $42.2 \pm 7.7$ & $25.4 \pm 4.7$ \\
\hline $\mathbf{G}_{16}$ & $23.7 \pm 5.3$ & $22.9 \pm 4.6$ & $38.1 \pm 3.3$ & $23.3 \pm 1.0$ & $55.4 \pm 5.4$ & $40.1 \pm 8.5$ \\
\hline $\mathbf{G}_{17}$ & $23.1 \pm 1.9$ & $16.5 \pm 2.4$ & $34.1 \pm 2.7$ & $32.1 \pm 7.1$ & $45.1 \pm 6.5$ & $28.9 \pm 3.7$ \\
\hline $\mathbf{G}_{18}$ & $93.0 \pm 2.3$ & $75.4 \pm 2.8$ & $45.5 \pm 3.0$ & $32.1 \pm 7.1$ & - & - \\
\hline 叶枯唑 ${ }^{b}$ & $74.1 \pm 3.3$ & $26.3 \pm 5.2$ & $40.5 \pm 2.0$ & $28.4 \pm 5.6$ & $41.7 \pm 4.7$ & $19.3 \pm 3.9$ \\
\hline 噻菌铜 ${ }^{b}$ & $40.0 \pm 3.0$ & $14.2 \pm 2.4$ & - & - & $30.7 \pm 4.9$ & $21.8 \pm 2.6$ \\
\hline
\end{tabular}

a. Average of three replicates; ${ }^{b}$ the commercial bactericide were used as positive control; "—" not detected. 
表 2 部分目标化合物对水稻白叶枯病菌的 $\mathrm{EC}_{50}$ 值 ${ }^{a}$

Table $2 \quad \mathrm{EC}_{50}$ values of some compounds against Xoo

\begin{tabular}{cccc}
\hline Compd. & $\mathrm{EC}_{50} /\left(\mu \mathrm{g} \cdot \mathrm{mL}^{-1}\right)$ & Regression equation & $R^{2}$ \\
\hline $\mathbf{G}_{\mathbf{1}}$ & $65.7 \pm 3.5$ & $y=0.840 x+3.473$ & 0.965 \\
$\mathbf{G}_{\mathbf{3}}$ & $87.6 \pm 0.3$ & $y=1.428 x+2.226$ & 0.995 \\
$\mathbf{G}_{\mathbf{5}}$ & $43.8 \pm 1.5$ & $y=1.452 x+2.616$ & 0.898 \\
$\mathbf{G}_{\mathbf{1 8}}$ & $5.34 \pm 0.2$ & $y=0.426 x+4.690$ & 0.978 \\
叶枯唑 $^{b}$ & $26.5 \pm 0.7$ & $y=1.497 x+2.182$ & 0.966 \\
噻菌铜 $^{b}$ & $69.3 \pm 2.1$ & $y=2.231 x+1.825$ & 0.944 \\
\hline
\end{tabular}

$\overline{a .}$ Average of three replicates; ${ }^{b}$ the commercial bactericide were used as positive control.

表 3 部分目标化合物对狝猴桃溃疡病菌的 $\mathrm{EC}_{50}$ 值 ${ }^{a}$

Table $3 \quad \mathrm{EC}_{50}$ values of some compounds against $P s a$

\begin{tabular}{cccc}
\hline Compd. & $\mathrm{EC}_{50} /\left(\mu \mathrm{g} \bullet \mathrm{mL}^{-1}\right)$ & Regression equation & $R^{2}$ \\
\hline $\mathbf{G}_{\mathbf{5}}$ & $78.2 \pm 2.5$ & $y=0.657 x+3.756$ & 0.987 \\
$\mathbf{G}_{\mathbf{7}}$ & $69.3 \pm 2.2$ & $y=1.520 x+2.202$ & 0.988 \\
$\mathbf{G}_{\mathbf{8}}$ & $61.4 \pm 0.9$ & $y=0.665 x+3.817$ & 0.937 \\
$\mathbf{G}_{\mathbf{1 0}}$ & $76.2 \pm 1.4$ & $y=1.322 \mathrm{x}+2.521$ & 0.954 \\
$\mathbf{G}_{\mathbf{1 2}}$ & $43.4 \pm 1.5$ & $y=1.436 x+2.648$ & 0.987 \\
$\mathbf{G}_{\mathbf{1 6}}$ & $121 \pm 1.3$ & $y=1.860 x+1.125$ & 0.943 \\
$\mathbf{G}_{\mathbf{1 8}}$ & $76.4 \pm 2.2$ & $y=0.582 x+3.904$ & 0.994 \\
叶枯唑 $^{b}$ & $135 \pm 1.3$ & $y=0.630 x+3.658$ & 0.921 \\
\hline
\end{tabular}

${ }^{a}$ Average of three replicates; ${ }^{b}$ the commercial bactericide were used as positive control.

表 4 部分目标化合物对柑橘溃疡病菌的 $\mathrm{EC}_{50}$ 值 ${ }^{a}$

Table $4 \quad \mathrm{EC}_{50}$ values of some compounds against Xac

\begin{tabular}{cccc}
\hline Compd. & $\mathrm{EC}_{50} /\left(\mu \mathrm{g} \bullet \mathrm{mL}^{-1}\right)$ & Regression equation & $R^{2}$ \\
\hline $\mathbf{G}_{\mathbf{5}}$ & $63.6 \pm 2.0$ & $y=1.701 x+1.932$ & 0.979 \\
$\mathbf{G}_{\mathbf{7}}$ & $67.0 \pm 0.2$ & $y=0.944 x+3.276$ & 0.966 \\
$\mathbf{G}_{\mathbf{1 0}}$ & $54.0 \pm 2.5$ & $y=1.348 x+2.665$ & 0.973 \\
$\mathbf{G}_{\mathbf{1 1}}$ & $70.7 \pm 0.5$ & $y=0.785 x+3.548$ & 0.993 \\
$\mathbf{G}_{\mathbf{1 3}}$ & $61.0 \pm 3.4$ & $y=0.890 x+3.411$ & 0.926 \\
$\mathbf{G}_{\mathbf{1 5}}$ & $89.8 \pm 0.9$ & $y=0.773 x+3.490$ & 0.999 \\
$\mathbf{G}_{\mathbf{1 6}}$ & $29.3 \pm 1.2$ & $y=0.643 x+4.057$ & 0.981 \\
$\mathbf{G}_{\mathbf{1 7}}$ & $67.0 \pm 4.3$ & $y=0.720 x+3.685$ & 0.936 \\
叶枯唑 $^{b}$ & $72.0 \pm 0.8$ & $y=1.322 x+2.545$ & 0.999 \\
\hline
\end{tabular}

$\overline{{ }^{a} \text { Average of three replicates; }{ }^{b} \text { the commercial bactericide were used as posi- }}$ tive control.

表 $1 \sim 4$ 抑菌活性测试表明, 大部分化合物都具有 一定的抑菌活性. 构效关系分析表明, 当取代基团为 $3-\mathrm{NO}_{2} \mathrm{C}_{6} \mathrm{H}_{4} 、 3,4-\mathrm{Cl}_{2} \mathrm{C}_{6} \mathrm{H}_{3} 、 4-\mathrm{CH}_{3} \mathrm{C}_{6} \mathrm{H}_{4} \mathrm{SO}_{2}$ 时, 目标化合 物对 $X o o$ 表现出很好的抑制率, 如化合物 $\mathbf{G}_{\mathbf{1}} 、 \mathbf{G}_{\mathbf{5}}$ 和 $\mathbf{G}_{\mathbf{1 8}}$ 的 $\mathrm{EC}_{50}$ 值分别为 $65.7 、 43.8$ 和 $5.34 \mu \mathrm{g} / \mathrm{mL}$, 优于对照药 剂噻菌铜; 当取代基团为 $3,4-\mathrm{Cl}_{2} \mathrm{C}_{6} \mathrm{H}_{3} 、 2-\mathrm{NO}_{2} \mathrm{C}_{6} \mathrm{H}_{4}$ 、 2- $\mathrm{CH}_{3} \mathrm{C}_{6} \mathrm{H}_{4}$ 及 $3,5-\mathrm{F}_{2} \mathrm{C}_{6} \mathrm{H}_{3}$ 时, 目标化合物对 $P s a$ 表现出 很好的抑制率, 如化合物 $\mathbf{G}_{\mathbf{5}} 、 \mathbf{G}_{\mathbf{7}} 、 \mathbf{G}_{\mathbf{8}}$ 和 $\mathbf{G}_{\mathbf{1 2}}$ 的 $\mathrm{EC}_{50}$ 值 分别为 78.2、69.3、61.4 和 $43.4 \mu \mathrm{g} / \mathrm{mL}$, 优于对照药剂 叶枯唑; 当取代基团为 $3-\mathrm{NO}_{2} \mathrm{C}_{6} \mathrm{H}_{4} 、 2-\mathrm{NO}_{2} \mathrm{C}_{6} \mathrm{H}_{4} 、 3-\mathrm{Cl}-$ 4- $\mathrm{FC}_{6} \mathrm{H}_{3}$ 以及 3-Cl-4- $\mathrm{FC}_{6} \mathrm{H}_{3}$ 时, 目标化合物对 $X a c$ 表现 出很好的抑制活性, 如化合物 $\mathbf{G}_{\mathbf{5}} 、 \mathbf{G}_{\mathbf{7}} 、 \mathbf{G}_{\mathbf{1 0}}$ 和 $\mathbf{G}_{\mathbf{1 6}}$ 的 $\mathrm{EC}_{50}$ 值分别为 63.6、67.0、54.0 和 $29.3 \mu \mathrm{g} / \mathrm{mL}$, 均优于阳性
对照药叶枯唑. 综上分析，化合物 $\mathbf{G}_{5}$ 对三种细菌的抑制 活性都高于阳性对照药, 化合物 $\mathbf{G}_{\mathbf{7}} 、 \mathbf{G}_{\mathbf{1 0}}$ 和 $\mathbf{G}_{\mathbf{1 6}}$ 对 $P s a$ 和 $X a c$ 两种细菌抑制活性均高于阳性对照药叶枯唑.

\section{2 结论}

以靛红酸酐、苯磺酰氯和苯肼盐酸盐等为起始原料 合成了 18 个新型含有腙结构单元的喹唑啉酮类化合物, 初步活性篮选表明, 大多数化合物对上述三种植物细菌 都有一定的抑制活性. 部分化合物如 $G_{18} 、 G_{12}$ 和 $G_{16}$ 分 别对水稻白叶枯细菌、猕猴桃溃疡病菌、柑橘溃疡病菌 的 $\mathrm{EC}_{50}$ 大大优于阳性对照药噻菌铜和叶枯唑, 其中化 合物 $\mathbf{G}_{\mathbf{5}}$ 对三种细菌都有较好的抑制活性, 具有一定的 广谱性.

\section{3 实验部分}

\section{1 仪器与试剂}

RE-52AA 型旋转蒸发仪(上海亚荣生化仪器厂) 、 MR-52AA 型恒温加热磁力搅拌器(德国 Heidolph)、 Sarforius 电子天平(德国赛多利斯集团)、X-4 型数字显 示显微熔点测定仪 (上海测微光电科技有限公司)、 Bruker Ascend-400 spectrometer, JEOL ECX-500 核磁共 振(TMS 为内标, 德国)、高分辨质谱仪(HRMS, 赛默飞 世尔科技有限公司). 所用试剂均为市售分析纯; 靛红 酸酐、对羟基苯甲醛、苯肼盐酸盐、氯乙酰氯等试剂均 购买于上海毕得医药科技有限公司.

\section{2 中间体及目标化合物的合成}

\subsection{1 中间体 $\mathbf{A} 、 \mathbf{B}$ 的合成}

中间体 A、B 的合成方法参考文献[14], 中间体 D、 $\mathbf{E}$ 的制备按照文献 $[15,16]$ 的方法合成.

\subsection{2中间体 $\mathbf{F}$ 的合成}

在 $50 \mathrm{~mL}$ 的圆底烧瓶中, 加入无水碳酸钾 $(5.82 \mathrm{~g}$, $42.2 \mathrm{mmol}$ )、对羟基苯甲醛(4.68 g, $38.4 \mathrm{mmol}$ )、DMF (50 $\mathrm{mL})$, 室温下搅拌 $15 \mathrm{~min}$, 加入中间体 $\mathbf{E}(8.00 \mathrm{~g}, 38.4$ $\mathrm{mmol})$ 和碘化钾 $(0.64 \mathrm{~g}, 3.8 \mathrm{mmol})$, 室温摚拌反应 $2 \mathrm{~h}$, 加入乙酸乙酯 $(60 \mathrm{~mL})$ 和质量分数为 $5 \%$ 的碳酸钠溶液 $(60 \mathrm{~mL})$, 搅拌 $30 \mathrm{~min}$, 静置分层, 有机层用水 $(30 \mathrm{~mL} \times$ $3)$ 洗涤, 无水硫酸钠干燥抽滤, 旋转蒸发除去溶剂, 再 用异丙醇重结晶得到中间体 4-((3-甲基-4-氧代-3,4-二氢 喹唑啉-2-基)甲氧基)苯甲醛. 黄色固体, 收率 $80 \%$. m.p. $156 \sim 157{ }^{\circ} \mathrm{C} ;{ }^{1} \mathrm{H}$ NMR (400 MHz, DMSO- $d_{6}$ ) $\delta: 9.90$ (s, $1 \mathrm{H}), 8.16(\mathrm{dd}, J=8.0,1.0 \mathrm{~Hz}, 1 \mathrm{H}), 7.90(\mathrm{~d}, J=8.8 \mathrm{~Hz}$, 2H), 7.82 (ddd, $J=8.6,7.2,1.5 \mathrm{~Hz}, 1 \mathrm{H}), 7.63$ (d, $J=7.7$ $\mathrm{Hz}, 1 \mathrm{H}), 7.59 \sim 7.53(\mathrm{~m}, 1 \mathrm{H}), 7.32(\mathrm{~d}, J=8.7 \mathrm{~Hz}, 2 \mathrm{H})$, $5.45(\mathrm{~s}, 2 \mathrm{H}), 3.62(\mathrm{~s}, 3 \mathrm{H})$; ESI-HRMS clacd for 
$\mathrm{C}_{17} \mathrm{H}_{15} \mathrm{~N}_{2} \mathrm{O}_{3}[\mathrm{M}+\mathrm{H}]^{+}$295.1077, found 295.1070.

3.2.3 目标化合物 $\mathbf{G}_{\mathbf{1}} \sim \mathbf{G}_{\mathbf{1 8}}$ 的合成

在 $50 \mathrm{~mL}$ 圆底烧瓶中依次加入中间体 F $(0.5 \mathrm{~g}, 1.7$ $\mathrm{mmol})$ 、甲苯 $(20 \mathrm{~mL})$ 、苯肼 $(0.365 \mathrm{~g}, 3.4 \mathrm{mmol})$ 、冰醋酸 $(0.01 \mathrm{~g}, 0.17 \mathrm{mmol})$, 室温搅拌反应 $30 \mathrm{~min}$, 加装分水 器, 升温至 $120{ }^{\circ} \mathrm{C}$ 回流分水反应, 薄层层析板(TLC)检 测反应, 反应 $24 \sim 26 \mathrm{~h}$, 反应结束后, 经抽滤、烘干得粗 产品, 粗产品用乙腈重结晶得到目标产物. 其它化合物 采用同样的合成方法.

3-甲基-2-(((4-((2-(3-硝基苯基)肼基)甲基)苯氧基) 甲基)喹唑啉-4(3H)-酮 $\left(\mathbf{G}_{\mathbf{1}}\right)$ : 黄色固体, 收率 77.2\%. m.p. $213.0 \sim 214.0{ }^{\circ} \mathrm{C} ;{ }^{1} \mathrm{H}$ NMR (500 MHz, DMSO- $\left.d_{6}\right) \delta$ : 10.67 (s, 1H), $8.12(\mathrm{dd}, J=8.0,1.3 \mathrm{~Hz}, 1 \mathrm{H}), 7.88(\mathrm{~s}, 1 \mathrm{H})$, $7.79(\mathrm{~d}, J=6.0 \mathrm{~Hz}, 2 \mathrm{H}), 7.63(\mathrm{~d}, J=8.8 \mathrm{~Hz}, 3 \mathrm{H}), 7.52(\mathrm{~m}$, $J=8.0,1.0 \mathrm{~Hz}, 2 \mathrm{H}), 7.43(\mathrm{t}, J=8.0 \mathrm{~Hz}, 1 \mathrm{H}), 7.40 \sim 7.35$ $(\mathrm{m}, 1 \mathrm{H}), 7.15(\mathrm{~s}, 1 \mathrm{H}), 7.13(\mathrm{~s}, 1 \mathrm{H}), 5.30(\mathrm{~s}, 2 \mathrm{H}), 3.59$ (s, $3 \mathrm{H}) ;{ }^{13} \mathrm{C}$ NMR (101 MHz, DMSO- $\left.d_{6}\right) \delta: 161.7,158.6$, $152.8,149.2,147.0,146.9,139.2,134.9,130.8,129.1$, $127.9,127.7,127.6,126.6,120.7,118.4,115.7,112.9$, 105.8, 69.2, 30.4; HRMS (AP-ESI) calcd for $\mathrm{C}_{23} \mathrm{H}_{20} \mathrm{~N}_{5} \mathrm{O}_{4}$ $[\mathrm{M}+\mathrm{H}]^{+} 430.1509$, found 430.1504 .

3 -甲基-2-(((4-)((2-(4-溴-2-氟苯基)肼基)甲基)苯氧 基)甲基)喹唑啉-4(3H)-酩 $\left(\mathbf{G}_{2}\right)$ ：白色固体，收率 57.4\%. m.p. $210.0 \sim 211.0{ }^{\circ} \mathrm{C} ;{ }^{1} \mathrm{H}$ NMR (500 MHz, DMSO- $\left.d_{6}\right) \delta$ : $10.23(\mathrm{~s}, 1 \mathrm{H}), 8.13(\mathrm{dd}, J=8.0,1.3 \mathrm{~Hz}, 1 \mathrm{H}), 8.06(\mathrm{~s}, 1 \mathrm{H})$, $7.82 \sim 7.77(\mathrm{~m}, 1 \mathrm{H}), 7.64(\mathrm{~d}, J=7.8 \mathrm{~Hz}, 1 \mathrm{H}), 7.59(\mathrm{~d}, J=$ $8.8 \mathrm{~Hz}, 2 \mathrm{H}), 7.55 \sim 7.51(\mathrm{~m}, 1 \mathrm{H}), 7.43 \sim 7.35(\mathrm{~m}, 2 \mathrm{H}), 7.22$ $(\mathrm{d}, J=8.7 \mathrm{~Hz}, 1 \mathrm{H}), 7.12(\mathrm{~d}, J=8.8 \mathrm{~Hz}, 2 \mathrm{H}), 5.30(\mathrm{~s}, 2 \mathrm{H})$, $3.59(\mathrm{~s}, 3 \mathrm{H}) ;{ }^{13} \mathrm{C}$ NMR (101 MHz, DMSO- $\left.d_{6}\right) \delta: 161.7$, $158.5,152.8,149.16\left(\mathrm{~d},{ }^{1} J_{\mathrm{C}-\mathrm{F}}=243.8 \mathrm{~Hz}\right), 146.9,140.4$, $134.9,133.7\left(\mathrm{~d},{ }^{3} J_{\mathrm{C}-\mathrm{F}}=10.0 \mathrm{~Hz}\right), 129.3,128.3\left(\mathrm{~d},{ }^{6} J_{\mathrm{C}-\mathrm{F}}=\right.$ $2.6 \mathrm{~Hz}), 127.9,127.8,127.6,126.7,120.7,118.5\left(\mathrm{~d},{ }^{2} J_{\mathrm{C}-\mathrm{F}}=\right.$ $20.9 \mathrm{~Hz}), 115.8,115.4\left(\mathrm{~d},{ }^{5} J_{\mathrm{C}-\mathrm{F}}=3.7 \mathrm{~Hz}\right), 108.2\left(\mathrm{~d},{ }^{4} J_{\mathrm{C}-\mathrm{F}}=\right.$ $8.8 \mathrm{~Hz}$ ), 69.2, 30.4; HRMS (AP-ESI) calcd for $\mathrm{C}_{23} \mathrm{H}_{19} \mathrm{BrFN}_{4} \mathrm{O}_{2}[\mathrm{M}+\mathrm{H}]^{+}$481.0669, found 481.0662.

3 -甲基-2-(((4-)((2-(4-氯-2-氟苯基)肼基)甲基)苯氧 基)甲基)喹唑啉-4(3H)-酮 $\left(\mathbf{G}_{3}\right)$ ：白色固体，收率 $63.3 \%$. m.p. $234.0 \sim 235.0{ }^{\circ} \mathrm{C} ;{ }^{1} \mathrm{H}$ NMR $\left(500 \mathrm{MHz}, \mathrm{DMSO}-d_{6}\right) \delta$ : $10.22(\mathrm{~s}, 1 \mathrm{H}), 8.15 \sim 8.10(\mathrm{~m}, 1 \mathrm{H}), 8.06(\mathrm{~s}, 1 \mathrm{H}), 7.82 \sim$ $7.77(\mathrm{~m}, 1 \mathrm{H}), 7.66 \sim 7.62(\mathrm{~m}, 1 \mathrm{H}), 7.59(\mathrm{~d}, J=8.9 \mathrm{~Hz}$, $2 \mathrm{H}), 7.55 \sim 7.50(\mathrm{~m}, 1 \mathrm{H}), 7.45(\mathrm{t}, J=8.9 \mathrm{~Hz}, 1 \mathrm{H}), 7.28$ $(\mathrm{dd}, J=11.7,2.3 \mathrm{~Hz}, 1 \mathrm{H}), 7.12$ (t, $J=7.8 \mathrm{~Hz}, 3 \mathrm{H}), 5.30$ (s, 2H), $3.59(\mathrm{~s}, 3 \mathrm{H}) ;{ }^{13} \mathrm{C}$ NMR (101 MHz, DMSO- $\left.d_{6}\right) \delta$ : $161.7,158.5,152.8,149.0\left(\mathrm{~d},{ }^{1} J_{\mathrm{C}-\mathrm{F}}=243.0 \mathrm{~Hz}\right), 146.9$, $134.9,133.4\left(\mathrm{~d},{ }^{3} J_{\mathrm{C}-\mathrm{F}}=9.8 \mathrm{~Hz}\right), 129.3,127.9,127.8,127.6$, $126.7,125.5\left(\mathrm{~d},{ }^{6} J_{\mathrm{C}-\mathrm{F}}=3.1 \mathrm{~Hz}\right), 121.3\left(\mathrm{~d},{ }^{4} J_{\mathrm{C}-\mathrm{F}}=9.3 \mathrm{~Hz}\right)$, $120.7,116.0\left(\mathrm{~d},{ }^{2} J_{\mathrm{C}-\mathrm{F}}=21.7 \mathrm{~Hz}\right), 115.7,115.0\left(\mathrm{~d},{ }^{5} J_{\mathrm{C}-\mathrm{F}}=\right.$ $4.1 \mathrm{~Hz}$ ), 69.2, 30.4; HRMS (AP-ESI) calcd for $\mathrm{C}_{23} \mathrm{H}_{19} \mathrm{ClF}-$ $\mathrm{N}_{4} \mathrm{O}_{2}[\mathrm{M}+\mathrm{H}]^{+}$437.1175, found 437.1167.

3 -甲基-2-(((4-((2-(3-氰基苯基)肼基)甲基)苯氧基) 甲基)喹唑啉-4(3H)-酮 $\left(\mathbf{G}_{4}\right)$ : 黄色固体, 收率 54.3\%. m.p. $202.0 \sim 203.0{ }^{\circ} \mathrm{C} ;{ }^{1} \mathrm{H}$ NMR (500 MHz, DMSO- $\left.d_{6}\right) \delta$ : 10.52 (s, 1H), $8.12(\mathrm{dd}, J=8.0,1.3 \mathrm{~Hz}, 1 \mathrm{H}), 7.85$ (s, 1H), $7.82 \sim 7.77(\mathrm{~m}, 1 \mathrm{H}), 7.63(\mathrm{~d}, J=8.7 \mathrm{~Hz}, 3 \mathrm{H}), 7.55 \sim 7.50$ (m, 1H), 7.35 (t, $J=7.9 \mathrm{~Hz}, 2 \mathrm{H}), 7.26(\mathrm{~d}, J=8.5 \mathrm{~Hz}, 1 \mathrm{H})$, 7.12 (d, $J=8.8 \mathrm{~Hz}, 2 \mathrm{H}), 7.09$ (d, $J=7.6 \mathrm{~Hz}, 1 \mathrm{H}), 5.30$ (s, $2 \mathrm{H}), 3.59(\mathrm{~s}, 3 \mathrm{H}) ;{ }^{13} \mathrm{C}$ NMR (101 MHz, DMSO- $\left.d_{6}\right) \delta$ : $161.7,158.5,152.8,146.9,146.5,138.9,134.9,130.8$, $129.3,128.0,127.8,127.6,126.7,122.0,120.7,119.7$, $116.9,115.7,114.5,112.3,69.2,30.5$; HRMS (AP-ESI) calcd for $\mathrm{C}_{24} \mathrm{H}_{20} \mathrm{~N}_{5} \mathrm{O}_{2}[\mathrm{M}+\mathrm{H}]^{+}$410.1611, found 410.1605 .

3-甲基-2-(((4-((2-(3,5-二氯苯基)肼基)甲基)苯氧基) 甲基)喹唑啉-4(3H)-酮 $\left(\mathbf{G}_{5}\right)$ ：白色固体，收率 65.2\%. m.p. $237.0 \sim 238.0{ }^{\circ} \mathrm{C} ;{ }^{1} \mathrm{H}$ NMR (500 MHz, DMSO- $\left.d_{6}\right) \delta$ : $10.55(\mathrm{~s}, 1 \mathrm{H}), 8.13(\mathrm{dd}, J=8.0,1.2 \mathrm{~Hz}, 1 \mathrm{H}), 7.84 \sim 7.78$ $(\mathrm{m}, 2 \mathrm{H}), 7.63(\mathrm{dd}, J=8.2,4.9 \mathrm{~Hz}, 3 \mathrm{H}), 7.53(\mathrm{t}, J=7.5 \mathrm{~Hz}$, $1 \mathrm{H}), 7.12(\mathrm{~d}, J=8.8 \mathrm{~Hz}, 2 \mathrm{H}), 6.96(\mathrm{~d}, J=1.8 \mathrm{~Hz}, 2 \mathrm{H}), 6.79$ $(\mathrm{t}, J=1.9 \mathrm{~Hz}, 1 \mathrm{H}), 5.30(\mathrm{~s}, 2 \mathrm{H}), 3.59(\mathrm{~s}, 3 \mathrm{H}) ;{ }^{13} \mathrm{C} \mathrm{NMR}$ $\left(101 \mathrm{MHz}, \mathrm{DMSO}-d_{6}\right) \delta: 161.7,158.7,152.8,148.1,146.9$, $139.7,135.1,134.9,129.3,129.0,128.6,128.1,127.8$, 127.6, 126.7, 125.8, 120.7, 117.4, 115.8, 110.4, 69.2, 30.4; HRMS (AP-ESI) calcd for $\mathrm{C}_{23} \mathrm{H}_{19} \mathrm{Cl}_{2} \mathrm{~N}_{4} \mathrm{O}_{2}[\mathrm{M}+\mathrm{H}]^{+}$ 453.0879, found 453.0874 .

3-甲基-2-(((4-((2-(2,5-二氯苯基)肼基)甲基)苯氧基) 甲基)喹唑啉-4(3H)-酮 $\left(\mathbf{G}_{6}\right)$ ：白色固体，收率 59.5\%. m.p. $211.0 \sim 212.0{ }^{\circ} \mathrm{C} ;{ }^{1} \mathrm{H}$ NMR (500 MHz, DMSO-d $\left.d_{6}\right) \delta: 9.95$ $(\mathrm{s}, 1 \mathrm{H}), 8.25(\mathrm{~s}, 1 \mathrm{H}), 8.13(\mathrm{dd}, J=8.0,1.3 \mathrm{~Hz}, 1 \mathrm{H}), 7.82 \sim$ $7.78(\mathrm{~m}, 1 \mathrm{H}), 7.63(\mathrm{t}, J=8.6 \mathrm{~Hz}, 3 \mathrm{H}), 7.56 \sim 7.52(\mathrm{~m}, 1 \mathrm{H})$, 7.46 (d, $J=2.5 \mathrm{~Hz}, 1 \mathrm{H}), 7.31$ (d, $J=8.5 \mathrm{~Hz}, 1 \mathrm{H}), 7.14$ (d, $J=8.8 \mathrm{~Hz}, 2 \mathrm{H}), 6.76(\mathrm{dd}, J=8.4,2.5 \mathrm{~Hz}, 1 \mathrm{H}), 5.31$ (s, $2 \mathrm{H}), 3.60(\mathrm{~s}, 3 \mathrm{H}) ;{ }^{13} \mathrm{C} \mathrm{NMR}\left(101 \mathrm{MHz}, \mathrm{DMSO}-d_{6}\right) \delta$ : $161.7,158.8,152.8,146.9,143.1,142.1,134.9,133.2$, $131.2,129.0,128.2,127.8,127.6,126.7,120.7,119.0$, 115.8, 115.0, 113.3, 69.2, 30.4; HRMS (AP-ESI) calcd for $\mathrm{C}_{23} \mathrm{H}_{19} \mathrm{Cl}_{2} \mathrm{~N}_{4} \mathrm{O}_{2}[\mathrm{M}+\mathrm{H}]^{+}$453.0879, found 453.0874.

3-甲基-2-(((4-((2-(2-硝基苯基)肼基)甲基)苯氧基) 甲基)喹唑啉-4(3H)-酮 $\left(\mathbf{G}_{7}\right)$ ：红色固体，收率 63.4\%. m.p. $180.0 \sim 181.0{ }^{\circ} \mathrm{C} ;{ }^{1} \mathrm{H}$ NMR $\left(500 \mathrm{MHz}, \mathrm{DMSO}-d_{6}\right) \delta$ : $11.19(\mathrm{~s}, 1 \mathrm{H}), 8.17 \sim 8.03(\mathrm{~m}, 3 \mathrm{H}), 7.97(\mathrm{~s}, 1 \mathrm{H}), 7.83 \sim$ 
$7.76(\mathrm{~m}, 1 \mathrm{H}), 7.65(\mathrm{dd}, J=17.8,8.6 \mathrm{~Hz}, 3 \mathrm{H}), 7.53(\mathrm{t}, J=$ $7.5 \mathrm{~Hz}, 1 \mathrm{H}), 7.14(\mathrm{t}, J=12.2 \mathrm{~Hz}, 4 \mathrm{H}), 5.32(\mathrm{~s}, 2 \mathrm{H}), 3.59$ (s, $3 \mathrm{H}) ;{ }^{13} \mathrm{C}$ NMR (101 MHz, DMSO- $\left.d_{6}\right) \delta: 161.7,159.1$, $152.8,151.1,146.9,142.1,138.5,134.9,128.7,128.5$, 127.8, 127.6, 126.7, 126.6, 120.7, 115.8, 111.5, 69.2, 30.4; HRMS (AP-ESI) calcd for $\mathrm{C}_{23} \mathrm{H}_{20} \mathrm{~N}_{5} \mathrm{O}_{4}[\mathrm{M}+\mathrm{H}]^{+}$ 430.1509, found 430.1507 .

3-甲基-2-(((4-((2-(2-甲基苯基)肼基)甲基)苯氧基) 甲基)喹唑啉-4(3H)-酮 $\left(\mathbf{G}_{8}\right)$ : 黄色固体, 收率 68.5\%. m.p. $214.0 \sim 215.0{ }^{\circ} \mathrm{C} ;{ }^{1} \mathrm{H}$ NMR (500 MHz, DMSO- $\left.d_{6}\right) \delta: 9.37$ (s, $1 \mathrm{H}), 8.15 \sim 8.11(\mathrm{~m}, 1 \mathrm{H}), 8.07(\mathrm{~s}, 1 \mathrm{H}), 7.82 \sim 7.78(\mathrm{~m}$, $1 \mathrm{H}), 7.65(\mathrm{~d}, J=7.6 \mathrm{~Hz}, 1 \mathrm{H}), 7.58(\mathrm{~d}, J=8.8 \mathrm{~Hz}, 2 \mathrm{H})$, $7.56 \sim 7.51(\mathrm{~m}, 1 \mathrm{H}), 7.36(\mathrm{~d}, J=7.6 \mathrm{~Hz}, 1 \mathrm{H}), 7.12(\mathrm{~d}, J=$ $8.8 \mathrm{~Hz}, 2 \mathrm{H}), 7.06(\mathrm{t}, J=7.7 \mathrm{~Hz}, 1 \mathrm{H}), 7.00(\mathrm{~d}, J=7.3 \mathrm{~Hz}$, $1 \mathrm{H}), 6.64(\mathrm{td}, J=7.3,1.1 \mathrm{~Hz}, 1 \mathrm{H}), 5.30(\mathrm{~s}, 2 \mathrm{H}), 3.60(\mathrm{~s}$, $3 \mathrm{H}), 2.17$ (s, 3H); ${ }^{13} \mathrm{C}$ NMR (101 MHz, DMSO- $\left.d_{6}\right) \delta$ : $161.7,158.1,152.9,146.9,143.8,138.3,134.9,130.7$, $130.0,127.8,127.6,127.5,127.1,126.7,120.9,120.7$, 118.9, 115.7, 112.3, 69.2, 30.5, 17.9; HRMS (AP-ESI) calcd for $\mathrm{C}_{24} \mathrm{H}_{23} \mathrm{~N}_{4} \mathrm{O}_{2}[\mathrm{M}+\mathrm{H}]^{+}$399.1815, found 399.1807.

3-甲基-2-(((4-((2-(3-三氟甲基苯基)肼基)甲基)苯氧 基)甲基)喹唑啉-4(3H)-酮 $\left(\mathbf{G}_{\mathbf{9}}\right)$ : 黄色固体, 收率 72.3\%. m.p. $212.0 \sim 213.0{ }^{\circ} \mathrm{C} ;{ }^{1} \mathrm{H}$ NMR (500 MHz, DMSO- $\left.d_{6}\right) \delta$ : $10.51(\mathrm{~s}, 1 \mathrm{H}), 8.13$ (dd, $J=8.0,1.4 \mathrm{~Hz}, 1 \mathrm{H}), 7.85(\mathrm{~s}, 1 \mathrm{H})$, $7.82 \sim 7.78(\mathrm{~m}, 1 \mathrm{H}), 7.65 \sim 7.61(\mathrm{~m}, 2 \mathrm{H}), 7.60(\mathrm{~s}, 1 \mathrm{H})$, $7.55 \sim 7.51(\mathrm{~m}, 1 \mathrm{H}), 7.38(\mathrm{t}, J=7.9 \mathrm{~Hz}, 1 \mathrm{H}), 7.28 \sim 7.22$ (m, 2H), $7.13(\mathrm{~d}, J=8.8 \mathrm{~Hz}, 2 \mathrm{H}), 6.99(\mathrm{~d}, J=7.6 \mathrm{~Hz}, 1 \mathrm{H})$, $5.30(\mathrm{~s}, 2 \mathrm{H}), 3.60(\mathrm{~s}, 3 \mathrm{H}) ;{ }^{13} \mathrm{C}$ NMR (101 MHz, DMSO- $\left.d_{6}\right)$ $\delta 161.7,158.5,152.8,146.9,146.5,138.6,134.9,130.6$, $130.4\left(\mathrm{q},{ }^{1} J_{\mathrm{C}-\mathrm{F}}=31.2 \mathrm{~Hz}\right), 129.4,127.8,127.8,127.6$, $126.7,124.9\left(\mathrm{q},{ }^{1} J_{\mathrm{C}-\mathrm{F}}=272.2 \mathrm{~Hz}\right), 120.7,115.8,114.8(\mathrm{~m}$, $\left.{ }^{1} J_{\mathrm{C}-\mathrm{F}}=20.8 \mathrm{~Hz}\right), 108.0\left(\mathrm{dd},{ }^{1} J_{\mathrm{C}-\mathrm{F}}=8.3,3.8 \mathrm{~Hz}\right), 69.2,30.4$; HRMS (AP-ESI) calcd for $\mathrm{C}_{24} \mathrm{H}_{20} \mathrm{ClF}_{3} \mathrm{~N}_{4} \mathrm{O}_{2}[\mathrm{M}+\mathrm{H}]^{+}$ 453.1532, found 453.1526 .

3-甲基-2-(((4-( (2-(3-氯-4-氟苯基)肼基)甲基)苯氧 基)甲基)喹唑啉-4(3H)-酮 $\left(\mathbf{G}_{10}\right)$ ：白色固体, 收率 $64.8 \%$. m.p. 206.0 207.0 ${ }^{\circ} \mathrm{C} ;{ }^{1} \mathrm{H}$ NMR (500 MHz, DMSO- $\left.d_{6}\right) \delta$ : $10.30(\mathrm{~s}, 1 \mathrm{H}), 7.82 \sim 7.77(\mathrm{~m}, 2 \mathrm{H}), 7.64(\mathrm{~d}, J=7.8 \mathrm{~Hz}$, $1 \mathrm{H}), 7.61(\mathrm{~s}, 1 \mathrm{H}), 7.59(\mathrm{~s}, 1 \mathrm{H}), 7.55 \sim 7.50(\mathrm{~m}, 1 \mathrm{H}), 7.20$ (t, $J=9.1 \mathrm{~Hz}, 1 \mathrm{H}), 7.12(\mathrm{~d}, J=1.4 \mathrm{~Hz}, 2 \mathrm{H}), 7.10(\mathrm{~s}, 1 \mathrm{H})$, $6.92 \sim 6.88(\mathrm{~m}, 1 \mathrm{H}), 5.29(\mathrm{~s}, 2 \mathrm{H}), 3.59(\mathrm{~s}, 3 \mathrm{H}) ;{ }^{13} \mathrm{C} \mathrm{NMR}$ $\left(101 \mathrm{MHz}\right.$, DMSO- $\left.d_{6}\right) \delta: 161.7,158.3,152.8,150.9(\mathrm{~d}$, $\left.{ }^{1} J_{\mathrm{C}-\mathrm{F}}=236.3 \mathrm{~Hz}\right), 146.9,143.4\left(\mathrm{~d},{ }^{5} J_{\mathrm{C}-\mathrm{F}}=1.9 \mathrm{~Hz}\right), 138.1$, $134.9,129.4,127.7,127.6,126.6,120.7,120.3\left(\mathrm{~d},{ }^{3} J_{\mathrm{C}-\mathrm{F}}=\right.$
$18.4 \mathrm{~Hz}), 117.7\left(\mathrm{~d},{ }^{2} J_{\mathrm{C}-\mathrm{F}}=21.9 \mathrm{~Hz}\right), 115.7,112.7,112.0(\mathrm{~d}$, ${ }^{4} J_{\mathrm{C}-\mathrm{F}}=6.4 \mathrm{~Hz}$ ), 69.2, 30.5; HRMS (AP-ESI) calcd for $\mathrm{C}_{23} \mathrm{H}_{19} \mathrm{ClFN}_{4} \mathrm{O}_{2}[\mathrm{M}+\mathrm{H}]^{+}$437.1175, found 437.1171.

3-甲基-2-(((4-((2-(3,5-二氟苯基)肼基)甲基)苯氧基) 甲基)喹唑啉-4 $(3 H)$-酮 $\left(\mathbf{G}_{11}\right)$ : 白色固体, 收率 $64.8 \%$. m.p. $206.0 \sim 207.0{ }^{\circ} \mathrm{C} ;{ }^{1} \mathrm{H}$ NMR (500 MHz, DMSO- $d_{6}$ ) $\delta$ : $10.58(\mathrm{~s}, 1 \mathrm{H}), 8.12(\mathrm{dd}, J=8.0,1.2 \mathrm{~Hz}, 1 \mathrm{H}), 7.84 \sim 7.76$ (m, 2H), 7.63 (dd, $J=8.1,5.9 \mathrm{~Hz}, 3 \mathrm{H}), 7.53(\mathrm{t}, J=7.5 \mathrm{~Hz}$, $1 \mathrm{H}), 7.12(\mathrm{~d}, J=8.8 \mathrm{~Hz}, 2 \mathrm{H}), 6.61(\mathrm{dd}, J=10.1,2.1 \mathrm{~Hz}$, $2 \mathrm{H}), 6.41(\mathrm{t}, J=9.4 \mathrm{~Hz}, 1 \mathrm{H}), 5.30(\mathrm{~s}, 2 \mathrm{H}), 3.59(\mathrm{~s}, 3 \mathrm{H}) ;{ }^{13} \mathrm{C}$ NMR (101 MHz, DMSO- $\left.d_{6}\right) \delta: 165.1\left(\mathrm{~d},{ }^{4} J_{\mathrm{C}-\mathrm{F}}=16.1 \mathrm{~Hz}\right)$, $162.7\left(\mathrm{~d},{ }^{5} J_{\mathrm{C}-\mathrm{F}}=16.1 \mathrm{~Hz}\right), 161.7,158.6,152.8,148.6(\mathrm{t}$, $\left.{ }^{3} J_{\mathrm{C}-\mathrm{F}}=13.9 \mathrm{~Hz}\right), 146.9,139.1,134.9,129.1,128.0,127.8$, 127.6, 126.7, 120.7, 115.7, $95.1\left(\mathrm{~d},{ }^{1} J_{\mathrm{C}-\mathrm{F}}=29.0 \mathrm{~Hz}\right), 93.3$ (t, $\left.{ }^{2} J_{\mathrm{C}-\mathrm{F}}=26.5 \mathrm{~Hz}\right), 69.2,30.4$; HRMS (AP-ESI) calcd for $\mathrm{C}_{23} \mathrm{H}_{19} \mathrm{~F}_{2} \mathrm{~N}_{4} \mathrm{O}_{2} \mathrm{~S}_{2}[\mathrm{M}+\mathrm{H}]^{+}$421.1470, found 421.1461 .

3-甲基-2-(((4-((2-(2,6-二氯苯基)肼基)甲基)苯氧基) 甲基)喹唑啉-4(3H)-酮 $\left(\mathbf{G}_{12}\right)$ ：褐色固体，收率 $68.5 \%$. m.p. $211.0 \sim 212.0{ }^{\circ} \mathrm{C} ;{ }^{1} \mathrm{H}$ NMR (500 MHz, DMSO- $\left.d_{6}\right) \delta$ : 9.30 (s, 1H), 8.12 (dd, $J=7.8,0.9 \mathrm{~Hz}, 1 \mathrm{H}), 7.96(\mathrm{~s}, 1 \mathrm{H})$, 7.78 (t, $J=7.6 \mathrm{~Hz}, 1 \mathrm{H}), 7.62(\mathrm{~d}, J=8.1 \mathrm{~Hz}, 1 \mathrm{H}), 7.54 \sim$ 7.49 (m, 3H), 7.40 (d, $J=8.1 \mathrm{~Hz}, 2 \mathrm{H}), 7.10(\mathrm{~d}, J=8.7 \mathrm{~Hz}$, $2 \mathrm{H}), 7.01(\mathrm{t}, J=8.0 \mathrm{~Hz}, 1 \mathrm{H}), 5.28(\mathrm{~s}, 2 \mathrm{H}), 3.58(\mathrm{~s}, 3 \mathrm{H}) ;{ }^{13} \mathrm{C}$ NMR $\left(101 \mathrm{MHz}\right.$, DMSO- $\left.d_{6}\right) \delta: 161.7,158.4,152.8,146.9$, $139.6,138.6,134.9,129.8,129.5,127.8,127.6,127.1$, 126.7, 124.7, 120.7, 115.7, 69.2, 30.4; HRMS (AP-ESI) calcd for $\mathrm{C}_{23} \mathrm{H}_{19} \mathrm{Cl}_{2} \mathrm{~N}_{4} \mathrm{O}_{2}[\mathrm{M}+\mathrm{H}]^{+}$453.0879, found 453.0876 .

3-甲基-2-(((4-((2-(2,5-二氟苯基)肼基)甲基)苯氧基) 甲基)喹唑啉-4(3H)-酮 $\left(\mathbf{G}_{13}\right)$ ：白色固体，收率 $54.8 \%$. m.p. $162.0 \sim 163.0{ }^{\circ} \mathrm{C} ;{ }^{1} \mathrm{H}$ NMR (500 MHz, DMSO- $\left.d_{6}\right) \delta$ : $10.31(\mathrm{~s}, 1 \mathrm{H}), 8.13(\mathrm{~d}, J=7.9 \mathrm{~Hz}, 1 \mathrm{H}), 8.08(\mathrm{~s}, 1 \mathrm{H}), 7.80$ (dd, $J=11.1,4.2 \mathrm{~Hz}, 1 \mathrm{H}), 7.63$ (dd, $J=11.0,8.5 \mathrm{~Hz}, 3 \mathrm{H}$ ), $7.53(\mathrm{t}, J=7.5 \mathrm{~Hz}, 1 \mathrm{H}), 7.21 \sim 7.15(\mathrm{~m}, 1 \mathrm{H}), 7.13(\mathrm{~d}, J=$ $8.7 \mathrm{~Hz}, 3 \mathrm{H}), 6.47$ (t, $J=8.4 \mathrm{~Hz}, 1 \mathrm{H}), 5.31$ (s, 2H), 3.60 (s, $3 \mathrm{H}) ;{ }^{13} \mathrm{C}$ NMR (101 MHz, DMSO- $\left.d_{6}\right) \delta: 161.7,159.8(\mathrm{~d}$, $\left.{ }^{1} J_{\mathrm{C}-\mathrm{F}}=237.5 \mathrm{~Hz}\right), 158.6,152.8,146.9,140.8,135.5(\mathrm{t}$, $\left.{ }^{2} J_{\mathrm{C}-\mathrm{F}}=12.0 \mathrm{~Hz}\right), 134.9,129.2,128.1,127.8,127.6,126.7$, $120.7,115.8,104.0\left(\mathrm{~d},{ }^{3} J_{\mathrm{C}-\mathrm{F}}=7.1 \mathrm{~Hz}\right), 103.7\left(\mathrm{~d},{ }^{1} J_{\mathrm{C}-\mathrm{F}}=7.5\right.$ $\mathrm{Hz}), 100.8\left(\mathrm{~d},{ }^{4} J_{\mathrm{C}-\mathrm{F}}=4.0 \mathrm{~Hz}\right), 100.5\left(\mathrm{~d},{ }^{5} J_{\mathrm{C}-\mathrm{F}}=3.0 \mathrm{~Hz}\right)$, 69.2, 30.4; HRMS (AP-ESI) calcd for $\mathrm{C}_{23} \mathrm{H}_{19} \mathrm{~F}_{2} \mathrm{~N}_{4} \mathrm{O}_{2}[\mathrm{M}+$ $\mathrm{H}]^{+}$421.1470, found 421.1464 .

3-甲基-2-(((4-((2-(2-三氟甲基苯基)肼基)甲基)苯氧 基)甲基)喹唑啉-4(3H)-酮 $\left(\mathbf{G}_{14}\right)$ : 黄色固体, 收率 58.1\%. m.p. $202.0 \sim 203.0{ }^{\circ} \mathrm{C} ;{ }^{1} \mathrm{H}$ NMR (500 MHz, DMSO- $\left.d_{6}\right) \delta$ : 
$9.58(\mathrm{~s}, 1 \mathrm{H}), 8.28(\mathrm{~s}, 1 \mathrm{H}), 8.13(\mathrm{dd}, J=8.0,1.4 \mathrm{~Hz}, 1 \mathrm{H})$, $7.82 \sim 7.78(\mathrm{~m}, 1 \mathrm{H}), 7.71(\mathrm{~d}, J=8.4 \mathrm{~Hz}, 1 \mathrm{H}), 7.64(\mathrm{~d}, J=$ $8.0 \mathrm{~Hz}, 1 \mathrm{H}), 7.61(\mathrm{~d}, J=8.8 \mathrm{~Hz}, 2 \mathrm{H}), 7.55 \sim 7.52(\mathrm{~m}, 1 \mathrm{H})$, $7.51 \sim 7.46(\mathrm{~m}, 2 \mathrm{H}), 7.14(\mathrm{~d}, J=8.8 \mathrm{~Hz}, 2 \mathrm{H}), 6.87$ (t, $J=$ $7.5 \mathrm{~Hz}, 1 \mathrm{H}), 5.31(\mathrm{~s}, 2 \mathrm{H}), 3.60(\mathrm{~s}, 3 \mathrm{H}) ;{ }^{13} \mathrm{C}$ NMR $(101$ MHz, DMSO- $\left.d_{6}\right) \delta: 161.7,158.7,152.8,146.9,143.2(\mathrm{~d}$, $\left.{ }^{3} J_{\mathrm{C}-\mathrm{F}}=1.1 \mathrm{~Hz}\right), 141.6,134.9,134.0,129.3,128.1,127.8$, 127.6, 126.7, $126.6\left(\mathrm{~d},{ }^{2} J_{\mathrm{C}-\mathrm{F}}=5.7 \mathrm{~Hz}\right), 120.7,118.7,115.8$, 115.1, $111.4\left(\mathrm{~d},{ }^{1} J_{\mathrm{C}-\mathrm{F}}=30.1 \mathrm{~Hz}\right), 69.2,30.5$; HRMS (AP-ESI) calcd for $\mathrm{C}_{24} \mathrm{H}_{20} \mathrm{~F}_{3} \mathrm{~N}_{4} \mathrm{O}_{2}[\mathrm{M}+\mathrm{H}]^{+}$453.1532, found 453.1527.

3-甲基-2-(((4-((2-(3,5-二甲基苯基)肼基)甲基)苯氧 基)甲基)喹唑啉-4(3H)-酮 $\left(\mathbf{G}_{15}\right)$ ：黄色固体，收率 $55.3 \%$. m.p. $209.0 \sim 210.0{ }^{\circ} \mathrm{C} ;{ }^{1} \mathrm{H}$ NMR (500 MHz, DMSO- $d_{6}$ ) $\delta$ : $10.01(\mathrm{~s}, 1 \mathrm{H}), 8.13(\mathrm{dd}, J=8.0,1.3 \mathrm{~Hz}, 1 \mathrm{H}), 7.86 \sim 7.69$ (m, 2H), $7.64(\mathrm{~d}, J=7.9 \mathrm{~Hz}, 1 \mathrm{H}), 7.59 \sim 7.48(\mathrm{~m}, 3 \mathrm{H})$, $7.10(\mathrm{~d}, J=8.8 \mathrm{~Hz}, 2 \mathrm{H}), 6.63(\mathrm{~s}, 2 \mathrm{H}), 6.33(\mathrm{~s}, 1 \mathrm{H}), 5.28$ (s, 2H), 3.59 (s, 3H), $2.17(\mathrm{~s}, 6 \mathrm{H}) ;{ }^{13} \mathrm{C}$ NMR $(101 \mathrm{MHz}$, DMSO- $\left.d_{6}\right) \delta: 161.7,158.0,152.9,146.9,145.8,138.4$, $136.3,134.9,130.0,127.8,127.6,127.4,126.7,120.8$, $120.7,115.7,110.2,69.3,30.5,21.7$; HRMS (AP-ESI) calcd for $\mathrm{C}_{25} \mathrm{H}_{25} \mathrm{~N}_{4} \mathrm{O}_{2}[\mathrm{M}+\mathrm{H}]^{+}$413.1972, found 413.1965 .

3-甲基-2-(((4-((2-(苯磺酰基)肼基)甲基)苯氧基)甲 基)喹唑啉-4(3H)-酮 $\left(\mathbf{G}_{16}\right)$ : 黄色固体, 收率 65.6\%. m.p. $188.0 \sim 189.0{ }^{\circ} \mathrm{C} ;{ }^{1} \mathrm{H}$ NMR (500 MHz, DMSO- $\left.d_{6}\right) \delta$ : 11.39 (s, $1 \mathrm{H}), 8.16(\mathrm{~d}, J=7.3 \mathrm{~Hz}, 1 \mathrm{H}), 7.94 \sim 7.87(\mathrm{~m}$, $3 \mathrm{H}), 7.83(\mathrm{~s}, 1 \mathrm{H}), 7.68 \sim 7.50(\mathrm{~m}, 7 \mathrm{H}), 7.14(\mathrm{~d}, J=8.7 \mathrm{~Hz}$, 2H), $5.33(\mathrm{~s}, 2 \mathrm{H}), 3.60(\mathrm{~s}, 3 \mathrm{H}) ;{ }^{13} \mathrm{C}$ NMR $(101 \mathrm{MHz}$, DMSO- $\left.d_{6}\right) \delta: 161.7,159.6,152.7,147.7,146.8,139.4$, $134.9,133.4,129.6,128.8,127.8,127.6,126.6,126.6$, 120.7, 115.7, 69.0, 30.3; HRMS (AP-ESI) calcd for $\mathrm{C}_{23} \mathrm{H}_{21} \mathrm{~N}_{4} \mathrm{O}_{4} \mathrm{~S}[\mathrm{M}+\mathrm{H}]^{+}$449.1278, found 449.1272.

3-甲基-2-(((4-((2-(对甲氧基苯磺酰基)肼基)甲基)苯 氧基)甲基)喹唑啉-4(3H)-酮 $\left(\mathbf{G}_{17}\right)$ ：褐色固体，收率 58.4\%. m.p. $90.0 \sim 91.0{ }^{\circ} \mathrm{C} ;{ }^{1} \mathrm{H}$ NMR (500 MHz, DMSO- $\left.d_{6}\right) \delta: 11.21(\mathrm{~s}, 1 \mathrm{H}), 8.16(\mathrm{dd}, J=8.0,1.2 \mathrm{~Hz}, 1 \mathrm{H})$, $7.87(\mathrm{~s}, 1 \mathrm{H}), 7.82(\mathrm{~s}, 1 \mathrm{H}), 7.80(\mathrm{~d}, J=1.7 \mathrm{~Hz}, 1 \mathrm{H}), 7.65(\mathrm{~d}$, $J=7.7 \mathrm{~Hz}, 1 \mathrm{H}), 7.59 \sim 7.51(\mathrm{~m}, 4 \mathrm{H}), 7.13(\mathrm{t}, J=8.5 \mathrm{~Hz}$, $4 \mathrm{H}), 5.33(\mathrm{~s}, 2 \mathrm{H}), 3.81(\mathrm{~s}, 3 \mathrm{H}), 3.60(\mathrm{~s}, 3 \mathrm{H}) ;{ }^{13} \mathrm{C} \mathrm{NMR}$ $\left(101 \mathrm{MHz}\right.$, DMSO- $\left.d_{6}\right) \delta: 163.0,161.7,159.6,152.7,147.1$, $146.8,134.9,131.0,129.8,128.7,127.8,127.6,126.6$, 120.7, 115.7, 114.8, 69.0, 56.1, 30.3; HRMS (AP-ESI) calcd for $\mathrm{C}_{24} \mathrm{H}_{23} \mathrm{~N}_{4} \mathrm{O}_{5} \mathrm{~S}[\mathrm{M}+\mathrm{H}]^{+}$479.1383, found 479.1373.
3-甲基-2-(((4-((2-(4-甲基苯磺酰基)肼基)甲基)苯氧 基)甲基)喹唑啉-4(3H)-酮 $\left(\mathbf{G}_{\mathbf{1 8}}\right)$ ：白色固体，收率 74.2\%. m.p. $183.0 \sim 184.0{ }^{\circ} \mathrm{C} ;{ }^{1} \mathrm{H}$ NMR (500 MHz, DMSO- $\left.d_{6}\right) \delta$ : 11.29 (s, 1H), 8.16 (d, $J=7.3 \mathrm{~Hz}, 1 \mathrm{H}), 7.87$ (s, 1H), 7.82 (t, $J=7.2 \mathrm{~Hz}, 1 \mathrm{H}), 7.76(\mathrm{~d}, J=8.1 \mathrm{~Hz}, 2 \mathrm{H}), 7.65$ (d, $J=8.1$ $\mathrm{Hz}, 2 \mathrm{H}), 7.55$ (dd, $J=16.4,8.2 \mathrm{~Hz}, 3 \mathrm{H}), 7.40$ (d, $J=8.0$ $\mathrm{Hz}, 2 \mathrm{H}), 7.14$ (d, $J=8.6 \mathrm{~Hz}, 2 \mathrm{H}), 5.33$ (s, 2H), 3.60 (s, $3 \mathrm{H}) ;{ }^{13} \mathrm{C}$ NMR (101 MHz, DMSO- $\left.d_{6}\right) \delta$ : 161.7, 159.6, $152.7,147.2,146.8,143.8,138.1,136.6,134.9,130.0$, $128.8,128.5,127.8,127.7,127.6,127.6,126.6,125.9$, 120.5, 115.7, 69.0, 30.3; HRMS (AP-ESI) calcd for $\mathrm{C}_{24} \mathrm{H}_{23} \mathrm{~N}_{4} \mathrm{O}_{4} \mathrm{~S}[\mathrm{M}+\mathrm{H}]^{+}$463.1434, found 463.1423.

\section{3 目标化合物的抑菌活性测试}

参考文献方法 ${ }^{[17]}$, 采用浊度法测试目标化合物在 200 和 $100 \mu \mathrm{g} / \mathrm{mL}$ 浓度下, 对柑橘溃疡病菌、猕猴桃溃 疡病菌和水稻白叶枯病菌等 3 种植物病菌的体外抑制活 性, 并对其中活性较好的化合物, 进行水稻白叶枯病菌 的 $\mathrm{EC}_{50}$ 测试. 使用 $\mathrm{NA}$ 固体培养基 $(10 \mathrm{~g}$ 葡萄糖、 $5 \mathrm{~g}$ 蛋 白胨、 $1 \mathrm{~g}$ 酵母菌、 $3 \mathrm{~g}$ 牛肉膏、 $15 \mathrm{~g}$ 琼脂和 $1000 \mathrm{~mL}$ 二 次水), 将柑橘溃疡病菌、猕猴桃溃疡病菌和水稻白叶枯 病菌活化, 放入 $28{ }^{\circ} \mathrm{C}$ 恒温培养箱中, 直到长出单菌落. 用接菌环选取适量的黄色单菌落, 放入含有 NB (10g 葡 萄糖、 $5 \mathrm{~g}$ 蛋白炼、 $1 \mathrm{~g}$ 酵母菌、 $3 \mathrm{~g}$ 牛肉膏和 $1000 \mathrm{~mL}$ 二次水)培养基的雉形瓶中, 在恒温摇床中振荡培养至 对数生长期备用.

将需要测试的药品配制到指定浓度, 分别取 $1 \mathrm{~mL}$ 加入到装有 $4 \mathrm{~mL} \mathrm{NB}$ 培养基的试管中, 再取 $40 \mu \mathrm{L}$ 含有 水稻白叶枯病菌、柑橘溃疡菌、烟草青枯菌的 NB 培养 基加入试管中, 在 $26 \sim 28{ }^{\circ} \mathrm{C} 、 180 \mathrm{r} / \mathrm{min}$ 恒温摇床振荡 培养, 待空白对照组 $\mathrm{NB}$ 液体培养基的 $\mathrm{OD}_{595}$ 值为 $0.6 \sim$ 0.8 , 在分光光度计上测定各个浓度的菌液 $\mathrm{OD}_{595}$ 值.

校正 $\mathrm{OD}_{595}$ =含菌培养基 $\mathrm{OD}_{595}$-无菌培养基 $\mathrm{OD}_{595}$ 抑制率 $(\%)=\left(\right.$ 校正后对照培养基菌液 $\mathrm{OD}_{595}-$ 校正含药培养基 $\mathrm{OD}_{595}$ )/校正后对照培养基菌液 $\mathrm{OD}_{595} \times 100 \%$.

辅助材料(Supporting Information) 化合物 G1 G18 的 ${ }^{1} \mathrm{H}$ NMR、 ${ }^{13} \mathrm{C}$ NMR 和 HRMS 谱图. 这些材料可以免 费从本刊网站(http://sioc-journal.cn/)上下载.

\section{References}

[1] Fan, Z. J.; Shi, J.; Luo, N.; Ding, M. H.; Bao, X. P. J. Agric. Food Chem. 2019, DOI: 10.1021 /acs.jafc.9b04733.

[2] Shao, L. H.; Gan, Y. Y.; Zhang, L. Q.; Wang, Z. C.; Ouyang, G. P. Chem. Bull. 2019, 82, 779 (in Chinese).

(邵利辉, 甘宜远，张丽琼，王贞超，欧阳贵平，化学通报，2019, 
$82,779$.

[3] Vande, A. P.; Elliott, V. L.; Chang, S.; Lpoez-ruiz, F. J.; Marcroft, S. J.; Idnurm, A. PloS One 2017, 12, e0188106.

[4] Zhao, X. Q.; Li, F.; Zhuang, W. P.; Xue, X. W.; Lian, Y. Y.; Fan, J. H.; Fang, D. S. Org. Process Res. Dev. 2010, 14, 346.

[5] Hao, M. C.; Yin, X. Z.; Wang, H.; Xu, L. Y.; Dong, J. H. Chin. J. Med. Chem. 2007, 17, 368 (in Chinese).

(郝明春, 殷学治, 王辉, 徐莉英, 董金华, 中国药物化学杂志, 2007, 17, 368.)

[6] Xu, P. Y.; Chen, Z. W. Chin. J. Synth. Chem. 2016, 24, 824 (in Chinese).

(徐盼云，陈志卫，合成化学, 2016, 24, 824.)

[7] Peng, J. B.; Geng, H. Q.; Wang, W.; Qi, X. X.; Ying, J.; Wu, X. F. J. Catal. 2018, 365, 10.

[8] Sojitra, N. A.; Dixit, R. B.; Patel, R. K.; Patel, J. P.; Dixit, B. C. J. Saudi Chem. Soc. 2016, 20, S29.

[9] Li, X. Q.; Gan, Y. Y.; Meng, J.; Li, W.; Qi, Y. Y.; Tian, K.; Ouyang, G. P.; Wang, Z. C. J. Heterocycl. Chem. 2018, 55, 1382.
[10] Kandile, N. G.; Mohamed, M. I.; Zaky, H.; Mohamed, H. M. Eur. J. Med. Chem. 2009, 44, 1989.

[11] Mughal, E. U.; Ayaz, M.; Hussain, Z.; Hasan, A.; Sadiq, A.; Riaz, M.; Malik, A.; Hussain, S.; Choudhary, M. Bioorg. Med. Chem. 2006, 14, 4704.

[12] Pang, F. H.; Dong, H. F.; Wang, X.; Li, F. Y.; Huang, L.; Lin, J. X.; Chem. Res. Appl. 2016, 26, 164.

[13] Le, Y.; Gan, Y. Y.; Fu, Y. H.; Liu, J. M.; Li, W.; Zou, X.; Zhou, Z. X.; Wang, Z. C.; Ouyang, G. P.; Yan, L. J. J. Enzyme Inhib. Med. Chem. 2019, 35, 555.

[14] Dey, R.; Kumar, P.; Jee, P. B. J. Org. Chem. 2018, 83, 5438.

[15] Hanusek, J.; Drabina, P.; Sedlák, M. S.; Rosa, P. J. Heterocycl. Chem. 2006, 43, 1281

[16] Kabri, Y.; Gellis, A.; Vanelle, P. Green Chem. 2009, 11, 201.

[17] Chen, Y.; Li, P.; Chen, M.; Su, S. J.; He, J.; Zhang, M.; Liu, L. W.; Chen, M. Chin. J. Org. Chem. 2019, 39, 2813 (in Chinese). (陈英, 李普, 陈梅, 苏时军, 贺军, 张敏, 柳立伟, 薛伟, 有机 化学, 2019, 39, 2813.) 